\title{
Analysis of systematic error in hadronic vacuum polarization contribution to muon $\mathrm{g-2}$
}

\section{Eigo Shintani*}

RIKEN Center for Computational Science, Kobe, Hyogo 650-0047, Japan

E-mail: shintani@riken.jp

\section{Yoshinobu Kuramashi}

Center for Computational Sciences, University of Tsukuba, Tsukuba, Ibaraki 305-8577, Japan

\begin{abstract}
We study systematic uncertainties in the lattice QCD computation of hadronic vacuum polarization (HVP) contribution to the muon $g-2$. In this proceedings we investigate two systematic effects; finite volume (FV) effect, mass correction and cutoff effect. We evaluate FV effect at the physical pion mass on two different volumes of $(5.4 \mathrm{fm})^{4}$ and $(10.8 \mathrm{fm})^{4}$ using PACS10 configurations at the same cut-off scale, and for the mass correction, we compare two different pion mass points, $m_{\pi}=135 \mathrm{MeV}$ and $146 \mathrm{MeV}$ pion, on large volume, $L / a=10.8 \mathrm{fm}$ and 8.1 $\mathrm{fm}$ respectively. Our results indicate that the chiral perturbation theory (ChPT) possibly underestimates FV effects in a long distance region, where multi-hadron state contributions including two-pion state become prominent. For the cutoff effect, we compare two forms of lattice vector operators, which are local and conserved (point-splitting) currents, by varying the cutoff scale on a more than $(10 \mathrm{fm})^{4}$ lattice at the physical point. The local-local current correlator shows smaller scaling violation than local-conserved one both in light and strange quark channels.
\end{abstract}

The 36th Annual International Symposium on Lattice Field Theory - LATTICE2018

22-28 July, 2018

Michigan State University, East Lansing, Michigan, USA.

\footnotetext{
${ }^{*}$ Speaker.
} 


\section{Introduction}

The muon anomalous magnetic moment $(g-2)_{\mu}$ has been a key observable for a proof of predictability of quantum field theory. We expect that there might be a sign of the new physics beyond SM (BSM) in the muon $g-2$ anomaly, which is 3-4 sigma deviation between the standard model (SM) prediction and the BNL experiment [1] suggested in 2004. To establish that, FermiLab and J-PARC $(g-2)_{\mu}$ experiment $[2,3]$ aiming at a factor $4-5$ improvement from the BNL experiment is forthcoming.

Lattice QCD (LQCD) is robust approach to estimate the HVP $(g-2)_{\mu}$, which is totally independent of the phenomenological estimate. This is a theoretical calculation based on the first principle of QCD, whereas the current precision of LQCD estimate is roughly an order of magnitude larger than the phenomenological one, and it then does not satisfy the accuracy to search the BSM physics (see a recent review [4] and reference therein). Recent LQCD calculations are carried out combined with an estimate of effective models, for instance, chiral perturbation theory (ChPT) [5] or Gounaris-Sakurai (GS) parametrization [6, 4], used to correct the FV effect on $(\sim 6$ $\mathrm{fm})^{3}$ boxes at a long distance. As pointed out in our previous study [7] it is essentially important to assess the FV effect in the LQCD computation of HVP $(g-2)_{\mu}$ employing the direct comparison between different volumes at the physical pion mass without any reliance on the effective models. In this proceedings, we perform more precise comparison with ChPT using an enlarged lattice more than $(10 \mathrm{fm})^{4}$ at the physical pion mass, which are the subset of PACS10 configurations [8] generated by the PACS Collaboration. We also investigate the lattice cutoff effect by comparing the results at two different cutoffs of $a^{-1}=2.33$ and $3.09 \mathrm{GeV}$ maintaining a physical volume with more than $(10 \mathrm{fm})^{4}$.

\section{Methodology}

In LQCD we perform a discretized coordinate-space summation of the correlator over the finite lattice volume defined as

$$
\begin{aligned}
{\left[a_{\mu}^{\mathrm{hvp}}\right]_{\mathrm{lat}}\left(r_{\mathrm{cut}}\right) } & =\frac{1}{2} \sum_{r / a=0}^{r_{\mathrm{cut}} / a-1}\left[C^{\Gamma \Gamma^{\prime}}(r) W_{r}(r)+C^{\Gamma \Gamma^{\prime}}(r+a) W_{r}(r+a)\right], \\
W_{r}(r) & =8 \alpha_{e}^{2} \int_{0}^{\infty} \frac{d \omega}{\omega} K_{E}\left(\omega^{2}\right)\left[\omega^{2} r^{2}-4 \sin ^{2}(\omega r / 2)\right]
\end{aligned}
$$

with

$$
C^{\Gamma \Gamma^{\prime}}(x)=\sum_{\mu}\left\langle V_{\mu}^{\Gamma}(x) V_{\mu}^{\Gamma^{\prime}}(0)\right\rangle,
$$

where $r$ denotes a distance from the source point. Here we define $V_{\mu}^{\mathrm{L}}(x)=Z_{V} \bar{q}(x) \gamma_{\mu} q(x)$, and $V_{\mu}^{\mathrm{C}}(x)=\left[\bar{q}(x+a \hat{\mu})\left(1+\gamma_{\mu}\right) U_{\mu}^{\dagger}(x) q(x)-\bar{q}(x)\left(1-\gamma_{\mu}\right) U_{\mu}(x) q(x+a \hat{\mu})\right] / 2$. This procedure introduces the systematic uncertainties due to not only the discretized summation but also the truncation at some finite distance $r_{\text {cut }}$.

As we will explain below, the lattice we used in this study is symmetric and its spatial/temporal extension is large enough to control the finite volume effect and the backward state propagation (BSP) effect investigated in [7]. We can perform the integral of Eq. (2.4) (summation of Eq. (2.6)) 
Table 1: Summary of lattice parameters for gauge field configurations used in this work. $\left({ }^{*}\right)$ On this ensemble we regard the rotated configurations according to rotational symmetry as the other configurations since there is less correlation between them. Originally 10 configurations are generated. $\left.{ }^{* *}\right)$ This is the gauge ensemble with extended temporal extension by copy of $64^{4}$ lattice.

\begin{tabular}{ccccccc}
\hline \hline & Refs. & $L / a[L]$ & $T / a[T]$ & $a^{-1}(\mathrm{GeV})$ & $m_{\pi}(\mathrm{MeV})$ & \#configs \\
\hline \multirow{2}{*}{ PACS10 } & {$[8]$} & $128[10.8 \mathrm{fm}]$ & $128[10.8 \mathrm{fm}]$ & $2.333(18)$ & 135 & 21 \\
& - & $160[10.3 \mathrm{fm}]$ & $160[10.3 \mathrm{fm}]$ & $3.087(30)$ & 135 & $40^{*}$ \\
\hline & {$[8,7]$} & $64[5.4 \mathrm{fm}]$ & $64[5.4 \mathrm{fm}]$ & $2.333(18)$ & 139 & 187 \\
& {$[7]$} & $64[5.4 \mathrm{fm}]$ & $128^{* *}[10.8 \mathrm{fm}]$ & $2.333(18)$ & 139 & 86 \\
\hline \hline
\end{tabular}

for each direction of $\rho=x, y, z, t$, which allows us to increase the statistics by four times without much computational cost.

\section{Configurations}

We use two subsets of PACS10 configurations, which are generated with the stout-smeared $O(a)$-improved Wilson-clover quark action and Iwasaki gauge action [11] on $128^{4}$ and $160^{4}$ lattices (spatial extension $L$ and temporal extension $T$ are symmetric) at $\beta=1.82$ and 2.00, respectively. In addition we also employ gauge field configurations on a $64^{4}$ and extended temporal extension $T / a=128$ lattice at $\beta=1.82$ for the FV study. Lattice parameters of these configuration sets are summarized in Table 1. We investigate the FV effects using the $128^{4}$ and $64^{4}$ lattices at the same lattice spacing, and the study of cutoff effects using the $128^{4}$ and $160^{4}$ lattices with the fixed physical volume.

The detailed description for configuration generation on the $128^{4}$ and $64^{4}$ lattices has been already given in Ref. [8]. Here we explain about the generation of the $160^{4}$ configurations. We employ the stout smearing parameter $\rho=0.1$, and the number of the smearing is six, which are the same as in the case of the $128^{4}$ lattice at $\beta=1.82$ [8]. We use a value of the improvement coefficient $c_{\mathrm{SW}}=1.02$ which is nonperturbatively determined by the Schrödinger functional (SF) scheme following Ref. [12]. The hopping parameters for the light (up-down) and strange quarks $\left(\kappa_{\mathrm{ud}}, \kappa_{\mathrm{S}}\right)=(0.125814,0.124925)$ are carefully adjusted to yield the physical pion and kaon masses $\left(m_{\pi}, m_{K}\right)=(135.0 \mathrm{MeV}, 497.6 \mathrm{MeV})$ with the use of the cutoff of $a^{-1}=3.09 \mathrm{GeV}(a=0.064 \mathrm{fm})$ determined from the $\Xi$ mass $m_{\Xi}=1.3148 \mathrm{GeV}$.

The renormalization constant $Z_{V}$ for the local vector current operator depends on the lattice cutoff scale. We obtain $Z_{V}=0.95153(76)$ at $a^{-1}=2.33 \mathrm{GeV}(\beta=1.82)$ with the Schrödinger (SF) functional scheme [13], and $Z_{V}=0.9673(19)$ at $a^{-1}=3.09 \mathrm{GeV}(\beta=2.00)$ from the nucleon structure function since the computation with SF functional scheme at $\beta=2.00$ has not been done yet. We, however, observe there is consistent result of $Z_{V}$ in our study of nucleon form factor [14]. The physical observables are measured at every 10 trajectories on $128^{4}$ and $64^{4}$, and every 5 trajectories on $160^{4}$. The statistical error is estimated by the jackknife analysis. We employ $1,4,5$ jackknife binsizes for the $128^{4}, 160^{4}, 64^{4}$ lattices, respectively, following the error analysis in [8]. 


\section{Numerical results}

\subsection{Finite volume effect}

From the right panel of Fig. 1, one can see that the integrand has a clear tendency of which the magnitude of integrand increase when $L / a$ increases from 64 to 128 . We also observe in the left panel of Fig. 1 the FV effect, which is defined as

$$
\Delta_{\mathrm{FV}}=\left[a_{\mu}^{\mathrm{hvp}}\left(r_{\mathrm{cut}}\right)\right]_{L / a=128}^{l}-\left[a_{\mu}^{\mathrm{hvp}}\left(r_{\mathrm{cut}}\right)\right]_{L / a=64}^{l},
$$

is same sign with the prediction of the leading order ChPT [15], while the magnitude is larger than the ChPT estimate. To clearly see its discrepancy, in Fig. 1 we plot the ratio of FV effect in LQCD and ChPT at each $r_{\text {cut }}$. One can see that LQCD data tends to become larger than ChPT prediction from $r \approx 1 \mathrm{fm}$, and this tendency does not change even if $r_{\text {cut }}$ increase while the statistical error becomes large.

Choosing $r_{\text {cut }} \simeq 2.6 \mathrm{fm}$, where the maximum point of window method in Ref. [16], the discrepancy of FV effect in the light quark sector of HVP $(g-2)_{\mu}$ from the leading order ChPT is estimated as

$$
\Delta_{\mathrm{FV}}^{\mathrm{lat}} / \Delta_{\mathrm{FV}}^{\mathrm{ChPT}}=1.74(71),
$$

on $L=5.4 \mathrm{fm}$ at the physical pion mass. The LQCD result indicates that the actual FV effect is possibly much larger than the ChPT prediction, which could impact on other recent LQCD results used ChPT and similar analytic formula to correct the FV effect of their HVP $(g-2)_{\mu}$ values on $\left(4-5 \mathrm{fm}^{3}\right)$ box. Our direct LQCD study suggests that there may need to take into account the further contributions of multi-hadron states, which is not captured in ChPT.
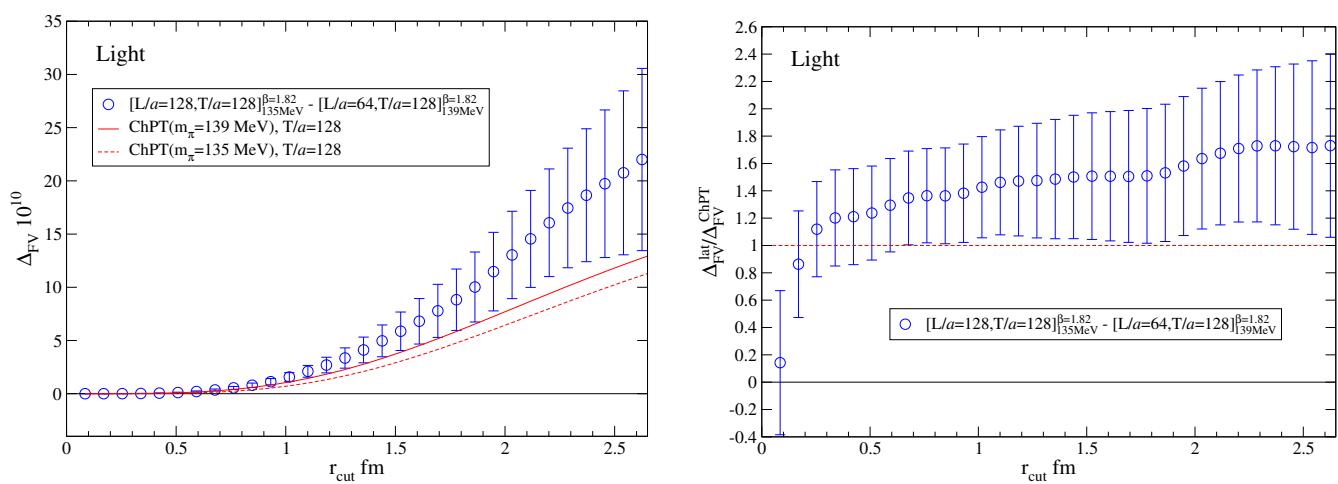

Figure 1: (Left) Integrated value up to $r_{\text {cut }}$ in light quark sector on $128^{4}$ and $64^{3} \times 128$ lattices. Results for $64^{3} \times 128$ lattice is at the simulation point of $m_{\pi}=139 \mathrm{MeV}$. Solid (dashed) curve denotes the ChPT prediction at the leading order of $\mathrm{FV}$ effect between spatial sizes $L / a=128$ and 64 on $T / a=128$ using and pion masses of $135 \mathrm{MeV}$ on $L / a=128$ and 139 (135) MeV on $L / a=64$. (Right) The ratio of FV effect in LQCD estimate and ChPT estimate at the leading order on $L=5.4 \mathrm{fm}$.

\subsection{Mass correction}

Compared to our previous study [7], in which we have estimated $\Delta_{\mathrm{FV} \text { on } 5.4 \mathrm{fm}}^{\text {lat }}=10(26) \times$ $10^{-10}$ in $146 \mathrm{MeV}$ pion from difference between $L=8.1 \mathrm{fm}$ and $L=5.4 \mathrm{fm}$ lattice result, besides 
two estimates are consistent within 1- $\sigma$ error, the central value is quite different. Such a discrepancy is possibly due to naive mass correction of $11 \mathrm{MeV}$ pion mass difference with linear ansatz. To confirm ambiguity of mass correction higher than $O\left(m_{\pi}\right)$ around physical pion mass, Figure 2 plots the difference between between $L=10.8 \mathrm{fm}$ in $135 \mathrm{MeV}$ pion and $L=8.1 \mathrm{fm}$ in $146 \mathrm{MeV}$ pion. One can see that there is significant discrepancy from ChPT prediction in IR regime. We expect that, since on $L=8.1 \mathrm{fm} \mathrm{FV}$ effect should be smaller than $L=5.4 \mathrm{fm}$, the mass dependence of $\left[a_{\mu}^{\text {hvp }}\right]^{l}$ around $m_{\pi} \simeq 140 \mathrm{MeV}$ is non-linearly enhanced by contribution beyond the (leading) ChPT even for $11 \mathrm{MeV}$ pion mass difference. This result indicates that we should be concerned with a reliance of ChPT for chiral extrapolation from unphysical pion [6]. Our work also provides an important suggestion for the other LQCD approach, which is using the simultaneous extrapolation into physical point from variant LQCD data-set relying on ChPT and related models (see for instance [4]).

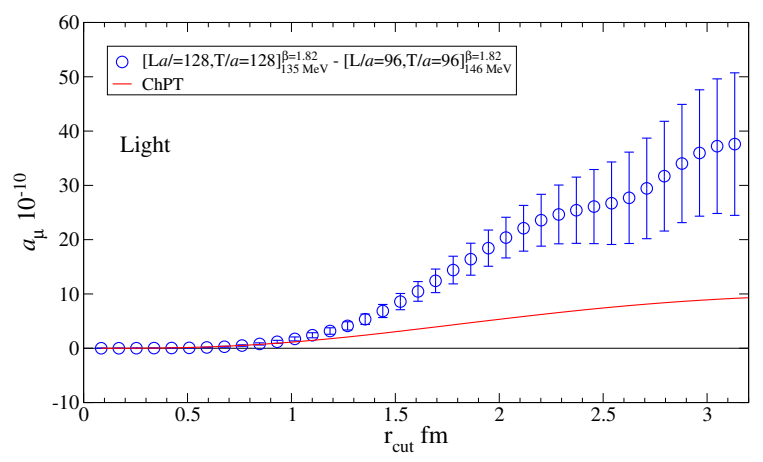

Figure 2: Difference of integrated value up to $r_{\text {cut }}$ on $128^{4}$ (with $135 \mathrm{MeV}$ pion) and $96^{4}$ (with $146 \mathrm{MeV}$ pion) in the light quark flavor. The solid line denotes an expectation in the leading order of chiral perturbation theory with the same lattice and pion mass.

\subsection{Cutoff effect}

We summarize the scaling properties for $\left[a_{\mu}^{\mathrm{hvp}}\right]_{\text {lat }}^{l},\left[a_{\mu}^{\mathrm{hvp}}\right]_{\text {lat }}^{s}$ and $\left[a_{\mu}^{\mathrm{hvp}}\right]_{\text {lat }}^{c}$ at two cutoff scales and their continuum extrapolations in Fig. 3, where the LQCD results at each cutoff scale are obtained by choosing $r_{\text {cut }} \approx 3.5 \mathrm{fm}$. One can see that (L,L) channel has rather small cutoff effect, which is not significant in the currently statistical precision, compared to $(\mathrm{C}, \mathrm{L})$ channel in the light and strange quark sectors. In our analysis, a constant fit of (L,L) channel is used for $\left[a_{\mu}^{\text {hvp }}\right]_{\text {lat }}^{l}$ and $\left[a_{\mu}^{\text {hvp }}\right]_{\text {lat }}^{s}$ to take the continuum extrapolation.

The systematic error is evaluated by taking the maximum difference between the central value, of which the constant fit is applied, and the linearly extrapolated values in the (L,L) channels with the ansatz of $\mathscr{O}(a)$ term including the error of lattice cutoff itself. The magnitude of the systematic error is comparable with that of the statistical one in the light and strange quark sectors. For the charm sector the bottom panel of Fig. 3 shows the large cutoff effect due to the $\mathscr{O}\left(a m_{c}\right)$ contribution even in the $(\mathrm{L}, \mathrm{L})$ channel. So we take the linearly extrapolated value in the $(\mathrm{L}, \mathrm{L})$ channel as the central value in the continuum limit. The systematic error is evaluated in the maximum difference between central value and extrapolated value in $(C, L)$ channel for which the higher order of cutoff effect than $O(a)$ is illustraited in the difference between $(\mathrm{C}, \mathrm{L})$ and $(\mathrm{L}, \mathrm{L})$ channel. In this 
case, the uncertainty of the cutoff effect is dominant error in the charm quark sector. For the total contribution of $\left[a_{\mu}^{\mathrm{hvp}}\right]_{\text {lat }}^{l}+\left[a_{\mu}^{\mathrm{hvp}}\right]_{\text {lat }}^{s}+\left[a_{\mu}^{\mathrm{hvp}}\right]_{\text {lat }}^{c}$, the uncertainty of light quark sector is still dominated.
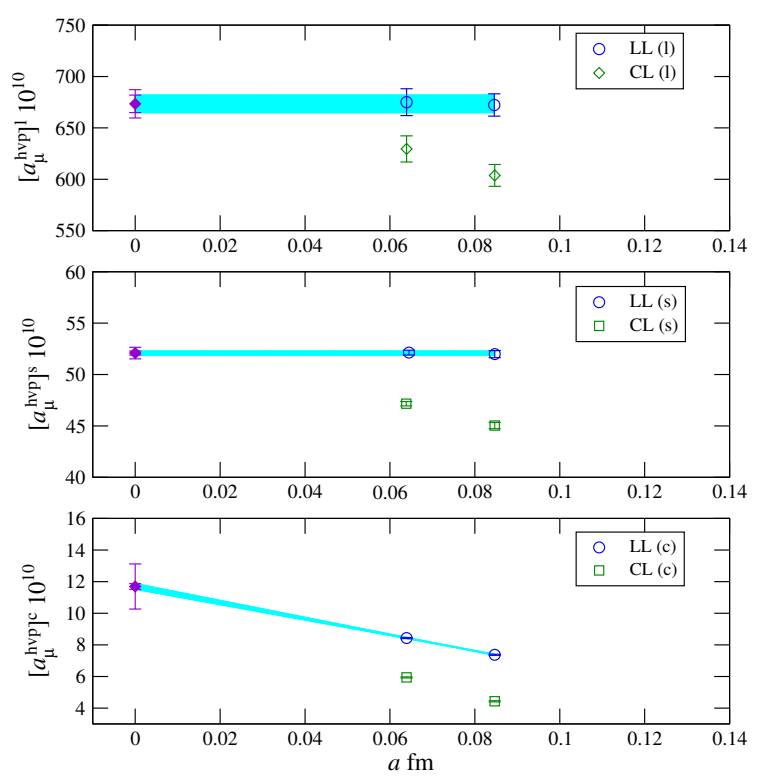

Figure 3: Cutoff dependence of $\left[a_{\mu}^{\mathrm{hvp}}\right]_{\text {lat }}^{l}\left(r_{\text {cut }}\right)$ (top), $\left[a_{\mu}^{\mathrm{hvp}}\right]_{\text {lat }}^{s}\left(r_{\text {cut }}\right)$ (middle) and $\left[a_{\mu}^{\mathrm{hvp}}\right]_{\text {lat }}^{c}\left(r_{\text {cut }}\right)$ (bottom) in $(\mathrm{L}, \mathrm{L})$ and $(\mathrm{C}, \mathrm{L})$ channels with $r_{\text {cut }} \approx 3.5 \mathrm{fm}$. The extrapolated result in the continuum limit (diamond) has two types of errors: inner one is statistical and outer one denotes the total error including the systematic error explained in the text.

\section{Summary}

We have studied the systematic uncertainties in the LQCD calculation of HVP $(g-2)_{\mu}$ on the PACS10 gauge configurations which have more than $(10 \mathrm{fm})^{4}$ box size at the physical point with two different lattice cutoffs. This study and previous work [7] are the direct LQCD calculation without use of any ansatz and reliance on any effective models. The optimized LQCD calculation of HVP on sufficiently large lattice size at the physical point allows us to access the deep IR regime where the contributions of multi-hadron states become manifest. Our study reveals that such contributions can not be completely captured by the chiral perturbation theory.

We plan to reduce the both statistical and systematic errors with additional computation including one more finer lattice, disconnected diagram, and QED effect in future. Here we will point out a possibility that the momentum-space integration scheme with $L>20 \mathrm{fm}$ can cover the peak position of kernel function in low $Q^{2}$ regime so that it can be complementary study to rigorously test LQCD scheme. We leave it to future work.

\section{Acknowledgments}

We originally develop the computation code based on Columbia Physics System(CPS) in 
which tuned OpenQCD system ${ }^{1}$ is embedded. Numerical calculations were performed on the K computer in RIKEN Center for Computational Science (CCS), Hokusai at Advanced Center for Computing and Communication (ACCC) in RIKEN, XC40 at YITP in Kyoto University, the Fujitsu PRIMERGY CX600M1/CX1640M1 (Oakforest-PACS) in the Information Technology Center, The University of Tokyo, and the computer facilities at Research Institute for Information Technology, Kyushu University. This computation is also supported by Interdisciplinary Computational Science Program No. xg18i015 in Tsukuba CCS, General use No. G18001 at ACCC, and resources of the K computer provided by the RIKEN-CCS through the HPCI System Research project (Project ID:hp180126).

\section{References}

[1] G. W. Bennett et al. [Muon g-2 Collaboration], Phys. Rev. Lett. 92, 161802 (2004) doi:10.1103/PhysRevLett.92.161802 [hep-ex/0401008].

[2] D. Flay [Muon g-2 Collaboration], PoS ICHEP 2016, 1075 (2017). doi:10.22323/1.282.1075

[3] K. Shimomura, Hyperfine Interact. 233, no. 1-3, 89 (2015). doi:10.1007/s10751-015-1159-3

[4] H. B. Meyer and H. Wittig, Prog. Part. Nucl. Phys. 104, 46 (2019) doi:10.1016/j.ppnp.2018.09.001 [arXiv: 1807.09370 [hep-lat]].

[5] M. Golterman, K. Maltman and S. Peris, Phys. Rev. D 95, no. 7, 074509 (2017) doi:10.1103/PhysRevD.95.074509 [arXiv:1701.08685 [hep-lat]].

[6] M. Della Morte et al., JHEP 1710, 020 (2017) doi:10.1007/JHEP10(2017)020 [arXiv:1705.01775 [hep-lat]].

[7] T. Izubuchi et al. [PACS Collaboration], Phys. Rev. D 98, no. 5, 054505 (2018) doi:10.1103/PhysRevD.98.054505 [arXiv:1805.04250 [hep-lat]].

[8] K.-I. Ishikawa et al. [PACS Collaboration], Phys. Rev. D 99, no. 1, 014504 (2019) doi:10.1103/PhysRevD.99.014504 [arXiv:1807.06237 [hep-lat]].

[9] D. Bernecker and H. B. Meyer, Eur. Phys. J. A 47, 148 (2011) doi:10.1140/epja/i2011-11148-6 [arXiv:1107.4388 [hep-lat]].

[10] H. B. Meyer, Eur. Phys. J. C 77, no. 9, 616 (2017) doi:10.1140/epjc/s10052-017-5200-3 [arXiv:1706.01139 [hep-lat]].

[11] Y. Iwasaki, arXiv:1111.7054 [hep-lat].

[12] Y. Taniguchi, PoS LATTICE 2012, 236 (2012) [arXiv:1303.0104 [hep-lat]].

[13] K.-I. Ishikawa et al. [PACS Collaboration], PoS LATTICE 2015, 271 (2016) doi:10.22323/1.251.0271 [arXiv:1511.08549 [hep-lat]].

[14] E. Shintani, K. I. Ishikawa, Y. Kuramashi, S. Sasaki and T. Yamazaki, Phys. Rev. D 99, no. 1, 014510 (2019) doi:10.1103/PhysRevD.99.014510 [arXiv:1811.07292 [hep-lat]].

[15] C. Aubin, T. Blum, P. Chau, M. Golterman, S. Peris and C. Tu, Phys. Rev. D 93, no. 5, 054508 (2016) doi:10.1103/PhysRevD.93.054508 [arXiv:1512.07555 [hep-lat]].

[16] T. Blum et al. [RBC and UKQCD Collaborations], Phys. Rev. Lett. 121, no. 2, 022003 (2018) doi:10.1103/PhysRevLett.121.022003 [arXiv:1801.07224 [hep-lat]].

\footnotetext{
${ }^{1}$ http://luscher.web.cern.ch/luscher/openQCD/
} 